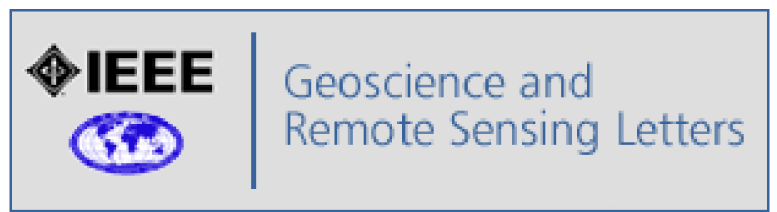

\title{
Implementation on Landsat Data of a Simple Cloud Mask Algorithm Developed for MODIS Land Bands
}

\begin{tabular}{|r|l|}
\hline Journal: & Geoscience and Remote Sensing Letters \\
\hline Manuscript ID: & Draft \\
\hline Manuscript Type: & Letters \\
\hline $\begin{array}{r}\text { Date Submitted by the } \\
\text { Author: }\end{array}$ & n/a \\
\hline Complete List of Authors: & $\begin{array}{l}\text { Oreopoulos, Lazaros; NASA-GSFC, Laboratory for Atmospheres } \\
\text { Wilson, Michael; UMBC, GEST } \\
\text { Varnai, Tamas; University of Maryland, Baltimore County, JCET }\end{array}$ \\
\hline Key Words: & Clouds, Satellites \\
\hline
\end{tabular}




\title{
Implementation on Landsat Data of a Simple Cloud Mask Algorithm Developed for MODIS Land Bands
}

\author{
by \\ Lazaros Oreopoulos ${ }^{1}$, Michael J. Wilson ${ }^{2,1}$ and Tamás Várnai ${ }^{3,1}$ \\ 1. Laboratory for Atmospheres, NASA-Goddard Space Flight Center, Code 613.2, \\ Greenbelt,MD 20771,Lazaros.Oreopoulos@nasa.gov \\ 2. Goddard Earth Sciences and Technology Center, University of Maryland \\ Baltimore County, Baltimore, MD 21250,Michael.J.Wilson-1@nasa.gov \\ 3. Joint Center for Earth Systems Technology, University of Maryland Baltimore \\ County, Baltimore, MD 21250,Tamas.Varnai@nasa.gov
}

Submitted to

IEEE Geoscience and Remote Sensing Letters

August 2010 


\begin{abstract}
This letter assesses the performance on Landsat-7 images of a modified version of a cloud masking algorithm originally developed for clear-sky compositing of Moderate Resolution Imaging Spectroradiometer (MODIS) images at northern mid-latitudes. While data from recent Landsat missions include measurements at thermal wavelengths, and such measurements are also planned for the next mission, thermal tests are not included in the suggested algorithm in its present form to maintain greater versatility and ease of use. To evaluate the masking algorithm we take advantage of the availability of manual (visual) cloud masks developed at USGS for the collection of Landsat scenes used here. As part of our evaluation we also include the Automated Cloud Cover Assesment (ACCA) algorithm that includes thermal tests and is used operationally by the Landsat-7 mission to provide scene cloud fractions, but no cloud masks. We show that the suggested algorithm can perform about as well as ACCA both in terms of scene cloud fraction and pixel-level cloud identification. Specifically, we find that the algorithm gives an error of $1.3 \%$ for the scene cloud fraction of 156 scenes, and a root mean square error of $7.2 \%$, while it agrees with the manual mask for $93 \%$ of the pixels, figures very similar to those from ACCA $(1.2 \%, 7.1 \%, 93.7 \%)$.
\end{abstract}

\title{
Index Terms
}

Clouds, Enhanced Thematic Mapper, Landsat, Moderate Resolution Imaging Spectroradiometer (MODIS), masking, Operational Land Imager (OLI), remote sensing, satellite, Thermal InfraRed Sensor (TIRS) 


\section{I. INTRODUCTION}

2 The presence of clouds in images acquired by the Landsat program is usually an undesirable, but 3 generally unavoidable fact. With the emphasis of the program being on land imaging, suspended

4 liquid/ice particles fully or partially obscure the desired observational target. Knowledge of 5 cloud amount in a Landsat scene and the location of clouds is therefore valuable information that

6 facilitates proper scene selection by Landsat data users, scene compositing from multiple scenes, 7 and scheduling of future acquisitions [1]. Presently, Landsat-7 images come with metadata that 8 provide the total cloud fraction of the scene (the fraction of cloudy over the total number of 9 pixels) as well as the cloud fraction in each of the four scene quadrants. These cloud "scores" are 10 generated by the Automated Cloud Cover Assessment (ACCA) algorithm [2]. Unfortunately, a 11 classification of individual pixels as either cloudy or cloud-free (i.e., a "cloud mask") is not 12 provided, forcing data users to perform their own cloud screening whenever their application 13 requires it. This will change for the next Landsat mission, the Landsat Data Continuity Mission 14 (LDCM), for which a cloud mask product is planned [3].

15 The purpose of this letter is to revisit a simple clear pixel detection algorithm developed for 16 MODIS 250/500 $\mathrm{m}$ land bands [4], unassisted by thermal data, and examine whether it can 17 provide pixel-level clear-cloudy sky discrimination for Landsat scenes at very small 18 computational cost. While we apply the algorithm in this paper only to Enhanced Thematic 19 Mapper Plus (ETM+) Landsat-7 data, it should be also applicable to historic Landsat-4 and 20 Landsat-5 data from the Thematic Mapper instrument, as well as data from the Operational Land 21 Imager (OLI) sensor of the upcoming LDCM. The cloud-shadow detection component, of the 22 original algorithm has not yet been fully validated in our Landsat implementation and will not be 23 further discussed in this paper. 


\section{II. THE CLOUD MASKING ALGORITHM}

25 The clear/cloud mask scheme introduced in [4] (hereafter "LTK scheme") is a simple threshold 26 scheme that uses only four MODIS $250 / 500 \mathrm{~m}$ resolution bands, specifically bands $1,2,3$, and 6 .

27 These bands have approximate spectral equivalents in the ETM+ instrument aboard Landsat-7

28 (Table 1). The LTK scheme threshold selection for surface type classification and cloud

29 detection is based on typical spectral signatures of five major pixel classes: non-vegetated land, 30 vegetated land, water, ice/snow and cloudy pixels, as depicted in Fig. 6 of [4]. The scheme 31 successively applies threshold tests to first classify non-vegetated pixels, followed by the 32 classification of ice/snow, water, and cloudy pixels. Any pixels not classified to any of the above 33 classes are assigned to the vegetated class. A flow chart presenting our modified LTK scheme is 34 provided in Fig. 1. After exhaustive testing of a variety of plausible adjustments to the LTK 35 thresholds to improve its performance, we settled on two threshold modifications in the last step 36 of the algorithm that separates cloud and vegetated pixel classes. These new thresholds resulted 37 in substantially better agreement between the cloud/clear masks from LTK and those from a 38 manual "truth" mask (discussed below) for a large collection of ETM+ scenes. Both the original 39 and modified LTK scheme threshold values are provided in the last box of the Fig. 1 flow chart, 40 and the performance of both variants of the scheme are contrasted in the next section. The 41 decrease of the ETM+ band 1 threshold (MODIS band 3) is consistent with the values of the 42 spectral reflectance plot for vegetated land shown in Fig. 6 of [4], which do not seem to exceed 43 0.1. However, the decrease of the band 5 threshold seems somewhat inconsistent with the 44 observed values of MODIS band 6 reflectances in the same plot, which seem to range between

450.1 and 0.18 . The fact that a lower value appears to work better for Landsat may be due to the 46 difference in spectral range and central wavelength location of the MODIS and Landsat bands. 
47 Numerous other threshold modifications also improved upon the original scheme, but none

48 worked as well as the two modifications that were eventually adopted. While we realize that a

49 cloud masking scheme developed for an instrument with similar spectral characteristics, but with

50 bands of different spectral widths, different spatial resolution, and off-nadir viewing capabilities,

51 should not necessarily translate perfectly to ETM+, we found nevertheless that in practice the

52 LTK scheme carries over quite well from MODIS to Landsat observations.

\section{III. ALGORITHM IMPLEMENTATION ON LANDSAT-7 SCENES}

55 The modified LTK scheme is applied to a collection of 156 Landsat scenes, a subset of the 212

56 scenes used by [2] to evaluate the performance of ACCA scene-averaged cloud fractions. The

57 criteria used to select the original dataset of 212 scenes is provided in [2]. These scenes are

58 approximately evenly apportioned among 9 latitude zones covering the entire globe. The present

59 subset of 156 consists of the scenes for which it was determined by USGS-EROS personnel that

60 a reliable cloud mask can be obtained. The manual mask was developed via a visual assessment

61 procedure [5]: Three experienced USGS imagery analysts performed manual assessment of the

62 scenes in [2]. 11 scenes were examined by all three in order to obtain the approximate error of

63 the procedure, which was found to be about $7 \%$ on average [5]. The process involved opening 64 each full resolution scene in Adobe Photoshop in a variety of RGB combinations, including 65 overlays of the (resampled) thermal band when necessary. The analysts then used appropriate 66 Photoshop image processing functions to isolate clouds. Two classes of clouds were identified:

67 thick and thin. Cloud pixels were labeled as thin if they were transparent but still visually 68 identifiable as clouds. For the purposes of this paper, no distinction is made between thin and 69 thick clouds in the quantitative metrics of the LTK scheme performance, but only when 
70 interpreting the results. Further quality evaluation of the visual mask was performed by an expert

71 remote sensing group at Boston University (BU) [6], which recommended that the collection of

72156 scenes be further reduced by 14 . Here we provide results for both the 156 and 142 scene

73 sets. As will be shown, both the ACCA and the LTK schemes agree better with the manual mask

74 for the smaller subset, a fact that seems to give further credence to the BU evaluation. In addition

75 to the USGS manual mask, we also have for these scenes the manually-determined cloud fraction

76 used as "truth" in [2]. The set of 156 contains 35 scenes from midlatitudes $\left(30^{\circ}-45^{\circ} \mathrm{N}\right.$ or $30^{\circ}$ -

$\left.7745^{\circ} \mathrm{S}\right), 41$ scenes from the subtropics $\left(15^{\circ}-30^{\circ} \mathrm{N}\right.$ or $\left.15^{\circ}-30^{\circ} \mathrm{S}\right), 21$ scenes from the tropics $\left(15^{\circ} \mathrm{S}-\right.$

$\left.7815^{\circ} \mathrm{N}\right), 33$ scenes from austral or boreal latitudes $\left(45^{\circ}-60^{\circ} \mathrm{S}\right.$ or $\left.45^{\circ}-60^{\circ} \mathrm{N}\right)$, and 26 scenes from the

79 polar regions $\left(60^{\circ}-90^{\circ} \mathrm{N}\right.$ or $\left.60^{\circ}-90^{\circ} \mathrm{S}\right)$. The $\mathrm{BU}$ group flagged as unreliable 7 polar visual masks

80 (4 from south and 3 from north), 1 austral mask, 2 tropical masks, 1 midlatitude south mask, and

813 midlatitude north masks. The fact that the original polar group of 44 scenes was reduced by the

82 combined manual mask screening re-evaluation of USGS and BU to 19 scenes should come as

83 no surprise, since cloud/ice/snow discrimination is very difficult even in visual image analysis.

84 Fig. 2 shows the outcome resulting from the original LTK scheme for a sample scene with

85 clear vegetated and non-vegetated land pixels, water pixels, and a fair amount of cloudy pixels.

86 The scheme appears to perform a reasonably good pixel classification and the clear/cloud mask,

87 although slightly worse than ACCA, makes the correct distinction between clear and cloudy

88 pixels more than $90 \%$ of the time.

89 The overall performance of the LTK scheme in terms of the "cloud score" (the cloud

90 fraction of the entire scene) can be seen in Fig. 3 for the 156 scene (top) and the 142 scene

91 (bottom) sets. The left panels correspond to the original LTK scheme and the right panels to our

92 modified version. ACCA results are included for comparison. The legends in each plot also 
93 contain summary metrics such as the overall bias in scene cloud fraction, the root mean square

94 error of the scene cloud fraction, and the number of "bad" scenes, defined here as scenes with

95 LTK or ACCA cloud fraction absolute differences from the manual mask ("cloud fraction 96 errors") that exceed $10 \%(=0.1$ when cloud fraction is measured in a scale from 0 to 1$)$.

97 Modifying the LTK scheme results in noticeable improvements which bring it on par, according 98 to our performance metrics, with the more complex ACCA scheme which includes thermal tests.

99 Using the same panel arrangement as in Fig. 3, Fig. 4 shows the performance of the 100 original and modified LTK schemes in terms of the percentage of pixels for which the algorithms 101 agree that a pixel is clear or cloudy ("mask agreement"). Again, the ACCA results are included 102 for comparison, allowing us to create a scatterplot of this metric where each scene is represented 103 by a point. Lines at the $80 \%$ agreement level are meant to isolate the poorer performers, 104 discussed further below. The benefits of modifying the LTK scheme are evident, as it more 105 closely approaches ACCA levels of performance. Note that that only 9 scenes have LTK mask 106 agreements below $80 \%$ (4 for the set of 142 ), but still always above $65 \%$.

107 If good cloud masking capabilities is the objective, then the mask agreement of Fig. 4 is a 108 better evaluator of the scheme's skill. If only the scene-average cloud fraction (score) is of 109 interest, and cancelling pixel misclassifications are tolerable, then the results of Fig. 3 are more 110 relevant. An obvious question is whether our collection of scenes includes cases with small 111 scene-average cloud fraction errors, but low mask agreement. Fig. 5 is a scatterplot of mask 112 agreement vs. cloud fraction error. As expected, there is a strong anticorrelation between the two 113 quantities. Scenes with small cloud fraction error usually exhibit high values of mask agreement.

114 With an arbitrary choice of $5 \%$ cloud fraction error and $80 \%$ mask agreement, only one scene 115 falls in the quadrant that indicates good cloud fraction estimates due to cancelling errors. 
116 We now examine why our modified LTK algorithm performs poorly for certain scenes,

117 either in terms of cloud fraction errors or mask agreements. We identified scenes belonging to

118 both of these categories of poor performance based on $10 \%$ (17 out of 156 scenes) and $80 \%$ (9

119 out of 156 scenes) thresholds, respectively, in order to investigate this question. 7 of the 9 scenes

120 that do not pass the $80 \%$ mask agreement threshold also belong to the subset of 17 scenes that do

121 not satisfy the $10 \%$ cloud fraction error criterion, so the number of unique "bad" scenes is 19 .

122 These 19 scenes have the following characteritics:

123 (a) 5 belong to the south pole latitude zone where cloud discrimination from ice and snow is 124 notoriously difficult.

125 (b) 7 exhibit greater than $10 \%$ cloud fraction error also between the manual USGS cloud scores 126 and the manual cloud scores of [2], which suggests that these scenes pose cloud identification 127 challenges even when visually inspecting RGB composites.

128 (c) 7 exhibit also poor ACCA performance (greater than $10 \%$ scene cloud fraction error); only 129 three of these belong to the 7 of category (b), yielding a total of 11 "difficult" scenes.

130 (d) For these 11 scenes, 6 have less than $80 \%$ pixel-level agreement for the ACCA algorithm as 131 well, and 7 were deemed to have unreliable USGS visual masks by the BU team (i.e. they 132 belong to the set of 156 , but not to the set of 142).

133 (e) 12 of the 19 scenes have high amounts of thin clouds, specifically a ratio of thin cloud pixels 134 to total number of cloudy pixels higher than the median value of 0.31 (derived from the 134 135 out of 156 scenes with non-zero cloudiness). 3 scenes have actually a ratio greater than 0.9 136 while only 2 have a ratio smaller than 0.1 . Thin cloud is very difficult to identify in land137 dominated scenes with a simple threshold algorithm relying only on solar bands. The LTK 138 scheme should therefore be used with caution for cloud masking when visual image 
139 inspection or other evidence (e.g., thermal band signatures) indicates the presence of thin 140 clouds.

\section{IV. SUMMARY AND CONCLUSIONS}

143 We have revisited a cloud/clear masking algorithm initially developed for MODIS clear-image

144 compositing and applied two threshold modifications that significantly improve its performance 145 when applied to a set of 156 Landsat scenes selected to cover the full range of Earth 146 geographical zones. The algorithm uses four Landsat solar bands that roughly correspond to the 147 MODIS bands of the original algorithm. We have found that despite its simplicity the algorithm 148 works quite well, giving a bias error of $1.3 \%$ for the scene cloud fraction of the 156 scenes, and a 149 root mean square error of $7.2 \%$. The algorithm agrees with the pixel classification (clear/cloudy) 150 of a manual (visual) mask for $93 \%$ of the pixels, on average. These performance metrics $(1.3 \%$, $1517.2 \%, 93.0 \%)$ are very close to those $(1.2 \%, 7.1 \%, 93.7 \%)$ of the more sophisticated Landsat-7 152 operation algorithm (ACCA), which also incorporates thermal band tests.

153 Two motivations for bypassing thermal tests are simplicity and speed. The modified LTK 154 scheme of this paper can be coded much easier by a non-expert than ACCA with its involved 155 "pass two" portion which, while helpful for re-classifying ambiguous pixels, has the drawback of 156 added complexity and greater execution time. Another reason to consider the scheme of this 157 paper, that may become relevant for future missions such as LDCM, is that cloud masking can 158 continue to operate even if no thermal data are available. This possibility is certainly not remote 159 for LDCM given the fact that solar and thermal sensing capabilities will be partitioned between 160 two instruments, OLI and TIRS, the latter of which has a shorter design life. While this modified 161 LTK scheme can be applied to historical Landsat data, its availability for future Landsat 
162 acquisitions is also important since it provides an extra cloud masking assessment opportunity

163 for whatever operational cloud mask algorithm is eventually adopted. The algorithm can also be

164 part of consensus cloud masks or masks with confidence level flags based on the degree of 165 agreement between an ensemble of distinct masking schemes.

166 Weaknesses of the modified LTK scheme that we have exposed in this work include its 167 limited ability to identify thin clouds and clouds over snowy or icy surfaces. Further work can 168 conceivably be undertaken to add a thermal component to the LTK algorithm and/or to add 169 threshold tests for the $1.38 \mu \mathrm{m}$ "thin cirrus" band of LDCM's OLI instrument. Both of these 170 elements have the potential to improve the LTK-based scheme significantly. Finally, it is 171 recognized that the testing of a masking algorithm on a collection of 156 scenes is not exhaustive 172 or conclusive, even if the scenes were selected to encompass most of the surface, solar geometry 173 and cloud type diversity encountered around the globe. Unfortunately, scheme evaluations that 174 involve manually generated masks cannot by nature be very extensive because the laborious 175 nature of visual pixel classification.

177 ACKNOWLEDGMENT

178 We would to thank Richard Irish (formerly of SSAI/NASA-GSFC) for providing the 212 179 Landsat-7 scenes used in [2] and with his manual cloud scores; Pat Scaramuzza (USGS/SGT) for 180 providing the visual cloud masks for the same dataset; and Curtis Woodcock (Boston University)

181 for his team's assessment of the USGS masks. Finally, we would like to acknowledge funding by 182 USGS under contract 06CRCN0022. 


\section{References}

184 [1] T. Arvidson, J. Gasch, and S. N. Goward, "Landsat 7's long term acquisition plan - An 185 innovative approach to building a global archive", Remote Sensing of Environment, vol. 78, no. $1861-2$, pp. 13-26, Oct. 2001.

187 [2] R. R. Irish, J. L. Barker, S. N. Goward, and T. Arvidson, "Characterization of the Landsat-7 188 ETM Automated Cloud-Cover Assessment (ACCA) Algorithm", Photogrammetric Engineering 189 \& Remote Sensing, vol. 72, no 10, pp. 1179-1188, Oct. 2006.

190 [3] T. Loveland, United States Geological Survey Earth Resources Observation Systems 191 (EROS) Data Center (EDC), Sioux Falls, SD, private communication, November 2009.

192 [4] Y. Luo, A. P. Trishchenko, and K. V. Khlopenkov, "Developing clear-sky, cloud and cloud 193 shadow mask for producing clear-sky composites at 250-meter spatial resolution for the seven 194 MODIS land bands over Canada and North America," Remote Sensing of Environment, vol. 112, 195 no. 12, pp. 4167-4185, Dec. 2008.

196 [5] P. Scaramuzza, United States Geological Survey Earth Resources Observation Systems 197 (EROS) Data Center (EDC), Sioux Falls, SD, private communication, November 2009.

198 [6] C. Woodcock, Boston University Boston, MA, private communication, April 2010. 199 


\section{List of Tables}

200

TABLE I

201

CORRESPONDENCE BETWEEN MODIS AND ETM+ BANDS

\begin{tabular}{|l|l|}
\hline \multicolumn{1}{|c|}{ MODIS } & \multicolumn{1}{c|}{ ETM+ } \\
\hline Band 1 (620-670 nm) & Band 3 (630-690 nm) \\
\hline Band 2 (841-876 nm) & Band 4 (750-900 nm) \\
\hline Band 3 (459-479 nm) & Band 1 (450-515 nm) \\
\hline Band 6 (1628-1652 nm) & Band 5 (1550-1750 nm) \\
\hline
\end{tabular}

202

203 Correspondence between the MODIS bands used in the original LTK scheme and the ETM+

204 bands used in this study for the modified LTK scheme.

205

206

List of Figures

207 Figure 1. Flow chart depicting the LTK clear/cloud masking part of the LTK scheme. The

208 numbers in parentheses in red are the original LTK thresholds for the reflectances of the 209 equivalent MODIS bands.

210 Figure 2. An example of applying the original LTK scheme on a Landsat-7 scene acquired on

211 April 22, 2001. (top) true color RGB image; (bottom) LTK pixel classification.

212 Figure 3. Comparison between manually-determined and cloud mask algorithm scene cloud

213 fractions (ACCA or LTK). Top row corresponds to the set of 156 Landsat scenes, and bottom

214 row to the reduced set of 142 scenes (see text for details). The left plots are for the original LTK

215 scheme, while the right plots show results after our modification. "Bad" in the legends refers to a

216 scene for which the cloud fraction error is greater than $10 \%(=0.1$ when cloud fraction is

217 measured in a scale from 0 to 1 ).

218 Figure 4. Comparison between LTK and ACCA mask agreement (in \%) for the original LTK 219 scheme (left panels) and the modified LTK scheme (right panels). The top row is for the set of 
1

2

3

4

5

6

7

8

9

10

11

12

13

14

15

16

17

18

19

20

21

22

23

24

25

26

27

28

29

30

31

32

33

34

35

36

37

38

39

40

41

42

43

44

45

46

47

48

49

50

51

52

53

54

55

56

57

58

59

60

220156 Landsat scenes while the bottom row is for the reduced set of 142 Landsat-7 scenes. "Bad"

221 in the legends refers to a scene for which the mask agreement is less than $80 \%$.

222 Figure 5. Scatterplot of the mask agreement of the modified LTK scheme against cloud fraction 223 error. The left lower quadrant identifies the number of scenes (one in this case) where a low 224 cloud fraction error $(<5 \%)$ can be achieved by cancellation of pixel misidentifications (as 225 measured by the \% mask agreement-less than $80 \%$ is considered poor performance). 226 


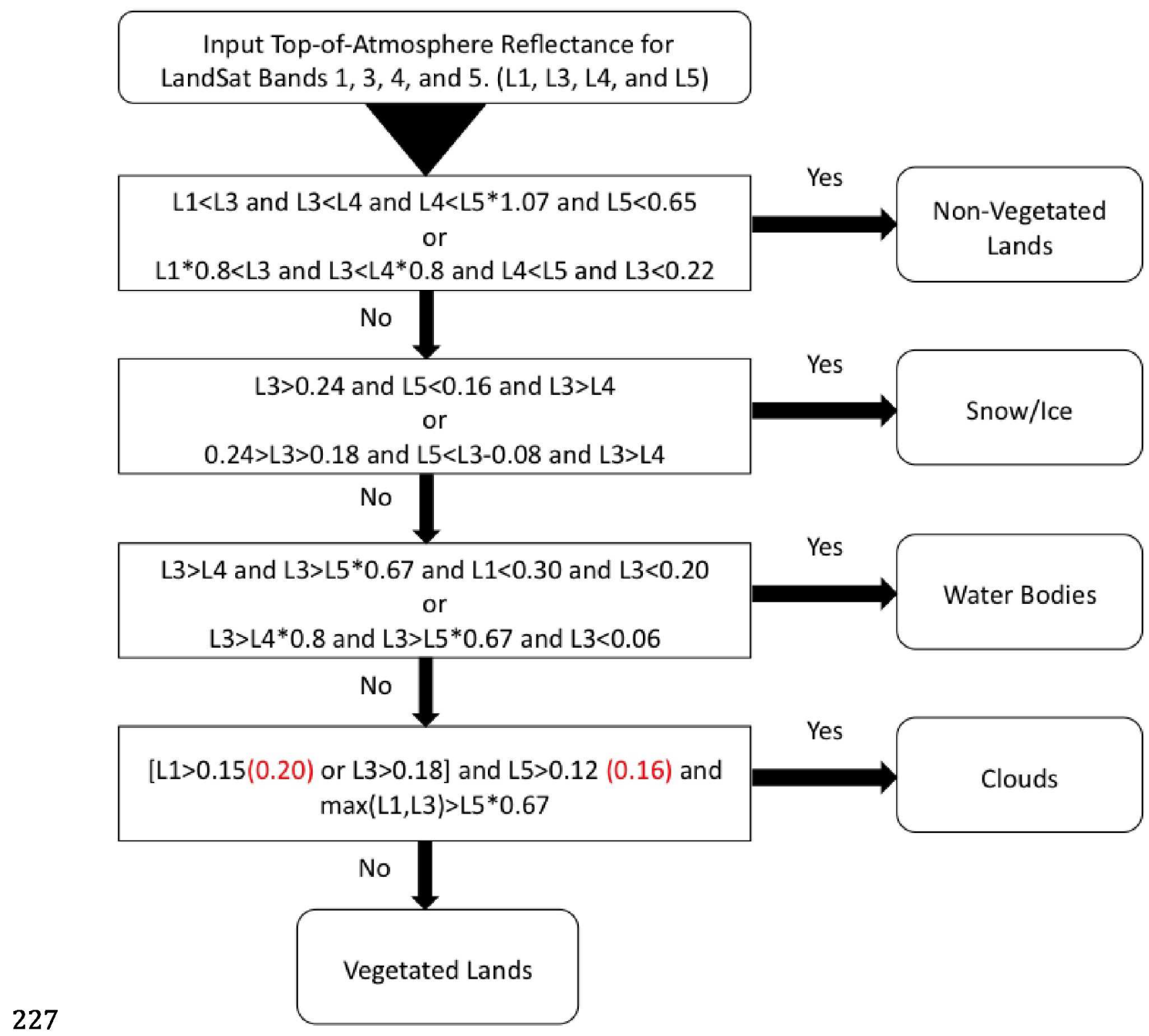

228 Figure 1. Flow chart depicting the LTK clear/cloud masking part of the LTK scheme. The 229 numbers in parentheses in red are the original LTK thresholds for the reflectances of the 230 equivalent MODIS bands. 


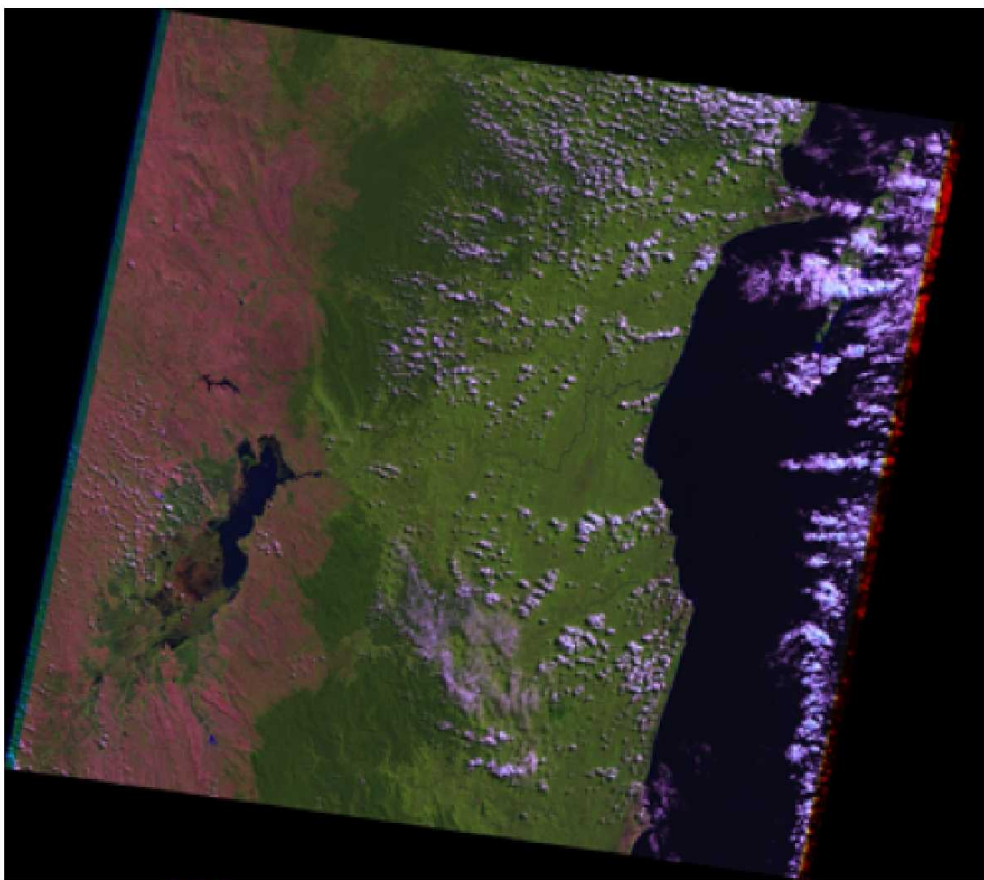

subtropical south

$$
\text { Path }=158
$$$$
\text { Row }=72
$$

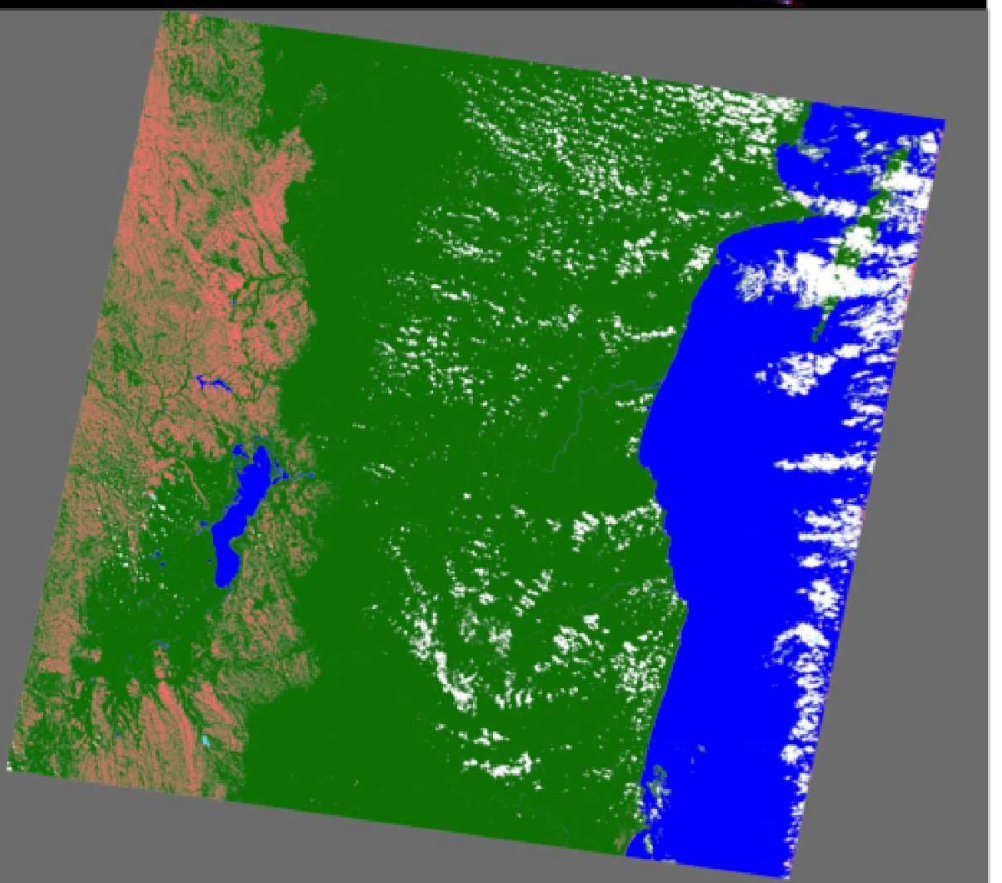

ACCA agreement $95.4 \%$

LTK agreement $91.5 \%$

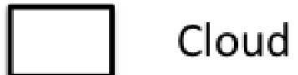

233 Figure 2. An example of applying the original LTK scheme on a Landsat-7 scene acquired on

234 April 22, 2001. (top) true color RGB image; (bottom) LTK pixel classification.

Non-Vegetated Lands

Snow/Ice
Water Bodies

\section{Vegetated Lands}





237 Figure 3. Comparison between manually-determined and cloud mask algorithm scene cloud 238 fractions (ACCA or LTK). Top row corresponds to the set of 156 Landsat scenes, and bottom 239 row to the reduced set of 142 scenes (see text for details). The left plots are for the original LTK 240 scheme, while the right plots show results after our modification. "Bad" in the legends refers to a 241 scene for which the cloud fraction error is greater than $10 \%(=0.1$ when cloud fraction is 242 measured in a scale from 0 to 1 ). 
1

2

3

4

5

6

10

11

12

13

14

15

16

17

18

19

20

21

\section{3}


246 Figure 4. Comparison between LTK and ACCA mask agreement (in \%) for the original LTK

247 scheme (left panels) and the modified LTK scheme (right panels). The top row is for the set of 248156 Landsat scenes while the bottom row is for the reduced set of 142 Landsat-7 scenes. "Bad" 249 in the legends refers to a scene for which the mask agreement is less than $80 \%$. 


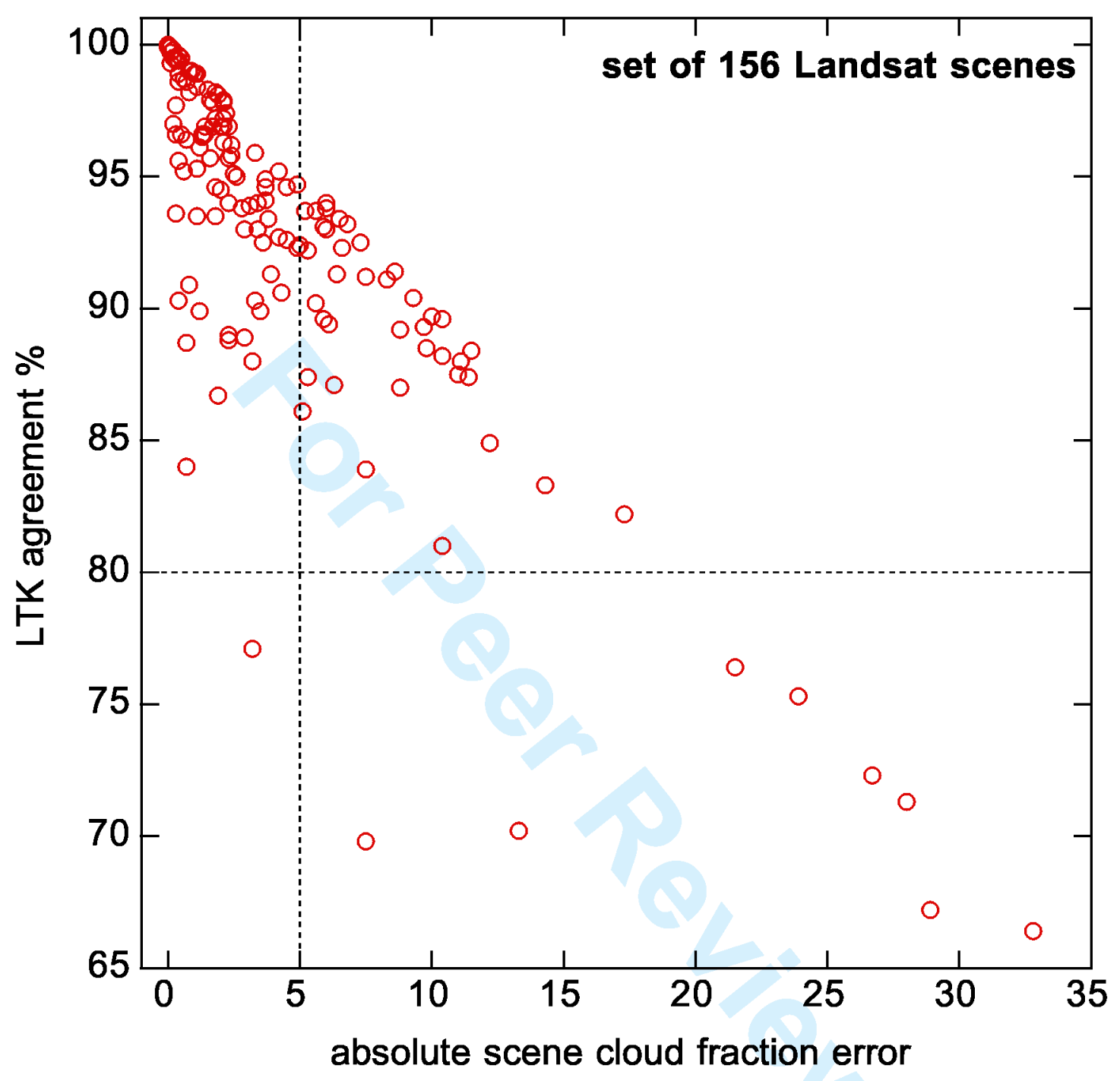

252 Figure 5. Scatterplot of mask agreement of the modified LTK scheme against cloud fraction 253 error. The left lower quadrant identifies the number of scenes (one in this case) where a low 254 cloud fraction error $(<5 \%)$ can be achieved by cancellation of pixel misidentifications (as 255 measured by the \% mask agreement-less than $80 \%$ is considered poor performance). 